\title{
Computed tomography-guided endoscopic recanalization of a completely obstructed
}

\section{rectal anastomosis}

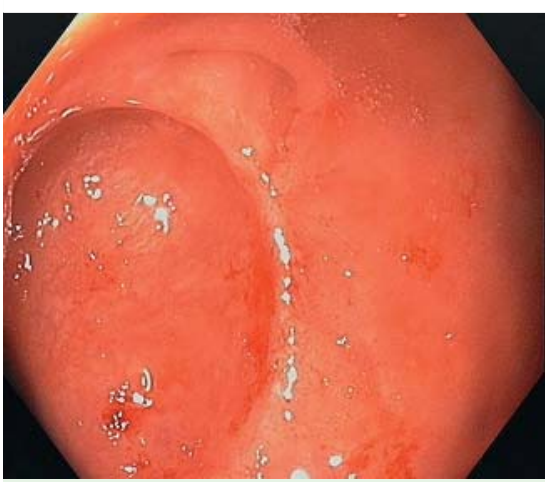

Fig. 1 Endoscopic view of the colorectal anastomosis from the rectum showing complete obstruction.

A 44-year-old man presented with complete obstruction of a colorectal anastomosis. He had undergone laparoscopic resection of the sigmoid colon 9 months previously for colonic perforation caused by endoscopic resection of a Peutz-Jeghers polyp. Surgical resection and reconstruction of the anastomosis, including diverging ileostomy, had been performed 6 months later because of anastomotic stricture and leakage. Closure of the ileostomy had been planned for 3 months later but high-pressure fluoroscopy showed no passage of contrast medium through the anastomosis and endoscopy confirmed complete obstruction with the former lumen being unidentifiable ( $\bullet$ Fig. 1 ). The anastomosis could not be reached endoscopically through the ileostomy because of peritoneal adhesions.

A computed tomography (CT) scan was performed and the colon was filled with air through the ileostomy. A gastroscope was advanced through the rectum and placed close to the anastomosis. The CT scan showed a membrane at the tip of the endoscope that was completely separating the descending colon and the rectum $(\bullet$ Fig. 2a). An incision of the membrane was performed under CT guidance using a needle-knife (OE11018N3; Endo-Flex, Voerde, Germany), and a guidewire was advanced through the incision. The CT scan confirmed the intracolonic position of the wire ( $\bullet$ Fig. $\mathbf{2} \mathbf{b}$ ) and dilation using a wire-guided balloon (M00558680; Boston Scientific, Natick, Massachusetts, USA) was

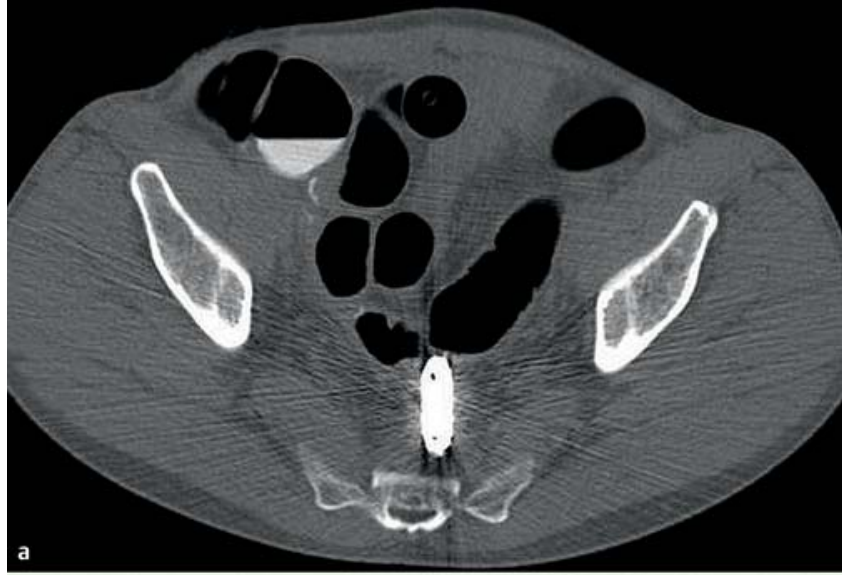

Fig. 2 Computed tomography (CT) scan showing: a the endoscope in the rectum and the air-filled descending colon, which are separated by a membrane; $\mathbf{b}$ the correctly positioned guidewire that had been advanced into the decending colon after incision of the membrane.

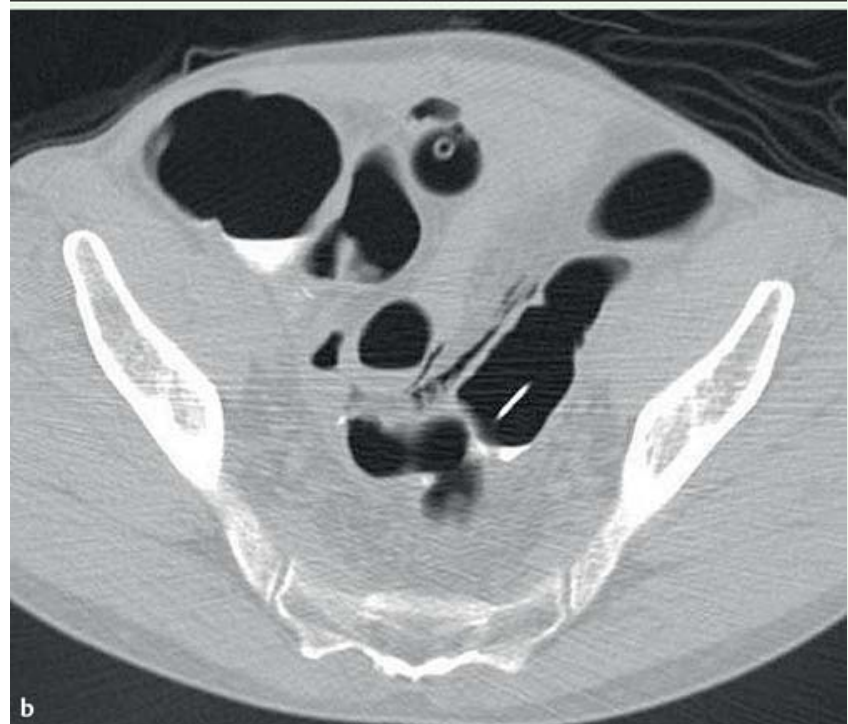

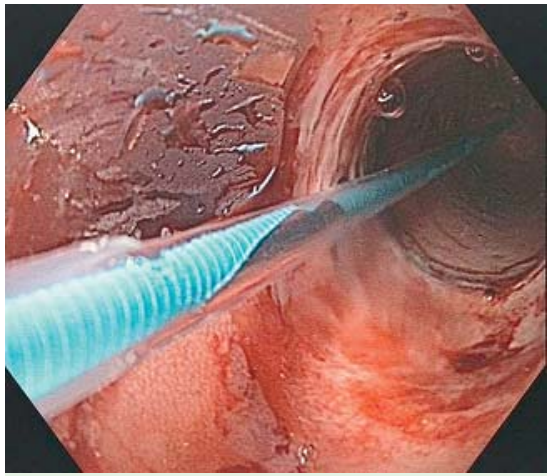

Fig. 3 Endoscopic view during balloon dilation after incision of the membrane and positioning of the guidewire under computed tomography (CT) guidance.

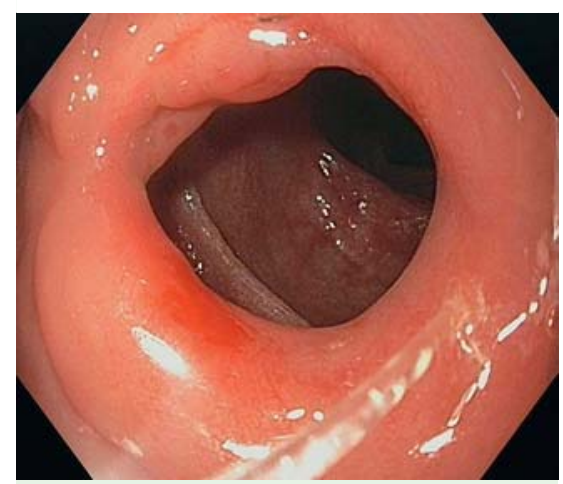

Fig. 4 Endoscopic view of the anastomosis 4 weeks after the endoscopic recanalization procedure. 
performed up to a diameter of $12 \mathrm{~mm}$ (๑ Fig. 3).

The ileostomy was closed surgically 4 weeks later. During the first four weeks after recanalization, endoscopic dilation was repeated weekly with $18-\mathrm{mm}$ balloons, by the end of which the stenosis had resolved completely ( $\bullet$ Fig.4). After 2 years, the patient remains free of symptoms.

Stricture of a colorectal anastomosis is a known complication and endoscopic dilation is the standard treatment. However, complete obstruction is rare and its treatment is not standardized. Case reports have described endoscopic approaches using different instruments, EUS-guided procedures, and rendezvous techniques [1-5]. In addition, CT guidance for endoscopic navigation should be considered to be helpful, especially when the anastomosis cannot be reached endoscopically from the proximal colon.

Endoscopy_UCTN_Code_TTT_1AQ_2AF

Competing interests: None
Andreas Probst ${ }^{1}$, Stefan Gölder ${ }^{1}$, Egbert Knöpfle ${ }^{2}$, Lukas Axt ${ }^{3}$, Helmut Messmann ${ }^{1}$

${ }^{1}$ Department of Gastroenterology, Klinikum Augsburg, Augsburg, Germany 2 Department of Radiology, Klinikum Augsburg, Augsburg, Germany

${ }^{3}$ Department of General, Visceral and Transplantation Surgery, Klinikum Augsburg, Augsburg, Germany

\section{References}

1 Kaushik N, Rubin J, McGrath K. Treatment of benign complete colonic anastomotic obstruction by using an endoscopic rendezvous technique. Gastrointest Endosc 2006; 63: $727-730$

2 De Lusong MA, Shah JN, Soetikno $R$ et al. Treatment of a completely obstructed colonic anastomotic stricture by using a prototype forward-array echoendoscope and facilitated by SpyGlass (with videos). Gastrointest Endosc 2008; 68: 988-992

3 Curcio G, Spada M, Di Francesco F et al. Completely obstructed colorectal anastomosis: electrosurgical endoscopic approach before balloon dilatation. World J Gastroenterol 2010; 16: $4751-4754$
4 Albertsmeier M, Rittler P, Hoffmann RT et al. Treatment of a completely obstructed colonic anastomotic stricture using a CT-guided endoscopic rendezvous technique. Endoscopy 2011; 43 (Suppl. 02): E5 -E6

5 Yazawa K, Morioka D, Matsumoto $C$ et al. Blunt penetration technique of a completely obstructed anastomosis after rectal resection: a case report. J Med Case Rep 2014; 8 : 236

Bibliography

DOI http://dx.doi.org/

10.1055/s-0034-1391131

Endoscopy 2015; 47: E32-E33

(C) Georg Thieme Verlag KG

Stuttgart · New York

ISSN 0013-726X

Corresponding author

Andreas Probst, MD

III. Medizinische Klinik

Klinikum Augsburg

Stenglinstrasse 2

86156 Augsburg

Germany

Fax: +49-821-4003331

andreas.probst@klinikum-augsburg.de 